\title{
The problem of raptor electrocution in Asia: case studies from Mongolia and China
}

\author{
ANDREW DIXON, ROLLER MAMING, AMARKHUU GUNGA, \\ GANKHUYAG PUREV-OCHIR and NYAMBAYAR BATBAYAR
}

\begin{abstract}
Summary
We report a large number of raptors electrocuted on recently erected electricity distribution lines in the open landscapes of the Mongolian steppe and Qinghai-Tibetan plateau, China. Upland Buzzards Buteo hemilasius and Saker Falcons Falco cherrug, characteristic raptors of these bioregions, were among those found to be electrocuted. Raptor electrocution was a consequence of poorly designed hardware configurations on anchor poles along surveyed lines on the Qinghai-Tibetan Plateau and, additionally, on line poles in the Mongolian steppe. The design flaws were upright pin-insulators on earthed crossarms and the use of jump wires that passed over crossarms via pin insulators on anchor poles. Targeted mitigation of anchor poles could significantly reduce the incidence of electrocution on the lines surveyed on the Qinghai-Tibetan Plateau, whilst all poles on the lines surveyed in the Mongolian steppe require remediation to make them safe for raptors. The Mongolian steppe and the Qinghai-Tibetan Plateau are bioregions that hold the largest breeding and wintering populations of the globally threatened Saker Falcon. The existing and growing network of dangerous electricity distribution lines in these regions may potentially impact the Saker Falcon population, thus we suggest that preventative and/or mitigation measures are implemented.
\end{abstract}

\section{Introduction}

Rapid global industrialisation over the last century has resulted in the development of vast networks of power distribution lines on all continents except Antarctica. The provision of electricity is integral to the economic and social development of every country and the health and social well-being of communities can be improved once they are connected to a reliable electricity supply (Chaurey et al. 2004), whilst the developed world has come to rely on an electricity supply for everyday existence. Given the importance of electricity for modern human society, the network of power distribution lines will continue to grow, especially in Asia and Africa.

Transmission lines, carrying electricity from a generation source, form the basis of an electricity grid and from this a network of distribution lines carry the electricity to places where it is needed. Transformers reduce the voltage between the transmission and distribution lines, and the distribution network carries a medium-voltage supply (generally 3 to $60 \mathrm{kV}$; APLIC 2006). Because the voltage is lower, the amount of insulation required is less than for transmission lines, which means that the energised conductor wires are closer to the crossarm from which they are suspended. Consequently, the proximity of the energized conductors to the line hardware and/or other phase conductors means that large birds, such as raptors, can become electrocuted by simultaneously contacting two phases, by contacting a single phase if their perching site is earthed or by arcing from a phase to an earthed, perched bird (Prinsen et al. 2011a).

Electrocution of birds on medium voltage electricity distribution lines is a widespread problem that has existed since power distribution lines were first erected in the late 19th century 
(Lehman et al. 1999). The problem has been recognized internationally and in North America and Europe steps have been taken to improve the safety of distribution lines (see references in APLIC 2006, Prinsen et al. 2011a, b), but the sheer scale of the electricity distribution network and the extent of mitigation required, mean that thousands of kilometres of power lines still pose an electrocution risk to birds. In Asia and Africa, the electricity distribution network is growing rapidly (Foster and Briceño-Garmendia 2010, Hammons 2011) and raptor electrocution is likely to be an increasing problem in these regions (Bevanger 1998, Boshoff et al. 2011, Angelov et al. 2013).

Relatively little information exists on raptor electrocution in Asia, though it is known to be responsible for high levels of raptor mortality in Kazakhstan (Lasch et al. 2010) and Asiatic Russia (Saltykov 2012). In recent years, studies have been undertaken in Mongolia to identify and highlight the problem of raptor electrocution (e.g. Gombobaatar et al. 2004, Harness et al. 2008, 2010, Amartuvshin and Gombobaatar 2012), where the problem is associated primarily with specific poles with complex wiring at junction points on older distribution lines with wooden poles, and more importantly, with recently installed lines using concrete poles and hardware manufactured in China (Dixon 2010), suggesting that the problem is likely to be widespread in China too. Little information exists on the scale or extent of raptor electrocution in China, but the problem has been documented in western China (Mei et al. 2008).

In this paper we report on raptor electrocutions at electricity distribution lines in two Mongolian provinces and in Qinghai, China. We describe the species found electrocuted on these lines at different times of year, electrocution events in relation to pole design and the efficacy of pre-existing mitigation measures. We suggest further mitigation for these lines and discuss the potential impact that electrocution may have on the globally threatened Saker Falcon Falco cherrug in these two remaining global strongholds for the species.

\section{Methods}

We undertook opportunistic surveys of electricity distribution lines in Mongolia and China during fieldwork for Saker Falcon conservation and management projects. The lines were selected for survey because carcasses of dead raptors were noted below poles; they do not represent a random selection of power distribution lines. We conducted whole line surveys rather than segmental surveys along each line.

\section{Line searches in Mongolia}

In Sukhbaatar Province we undertook three searches along a three-phase, $15 \mathrm{kV}$ electricity distribution line running $56 \mathrm{~km}$ from the district centre of Uulbayan to the district centre of Monkhkhaan: spring (12-13 May 2009), autumn (7 September 2011) and winter (8 March 2012). The line was constructed in 2004 and crosses open, undulating and treeless landscape in the central Mongolian steppe with few potential above-ground perching sites for raptors. All poles were constructed of reinforced concrete and the line comprised 493 standard 'line' poles and 35 'anchor' poles (Figure $\mathrm{rb}, \mathrm{c} \& \mathrm{~d}$ ). This line has been surveyed previously for electrocuted raptors in 2007 and 2009 (Harness et al. 2008, 2010) and a number of poles had been fitted with two or four perch-deterrent spikes in an attempt to reduce electrocution risk for birds of prey. The placement of these spikes differed in that when only two were used they were placed centrally on the crossarm but when four were used the outermost spikes were closer to the phase wire (Figure 1 e \& f). We have termed these two configurations as non-functional and functional respectively, because spikes placed centrally cannot prevent birds from perching next to the phases at the ends of the crossarms.

In Dundgovi Province, we undertook a single search along a three-phase, $15 \mathrm{kV}$ distribution line running $71 \mathrm{~km}$ from the district centre of Olziit to the district centre of Khuld on 11 June 2010. The line was erected in 2006 and crosses open, undulating and treeless landscape in the central Mongolian steppe. The Olziit-Khuld line comprised 629 poles and most had been fitted with two 

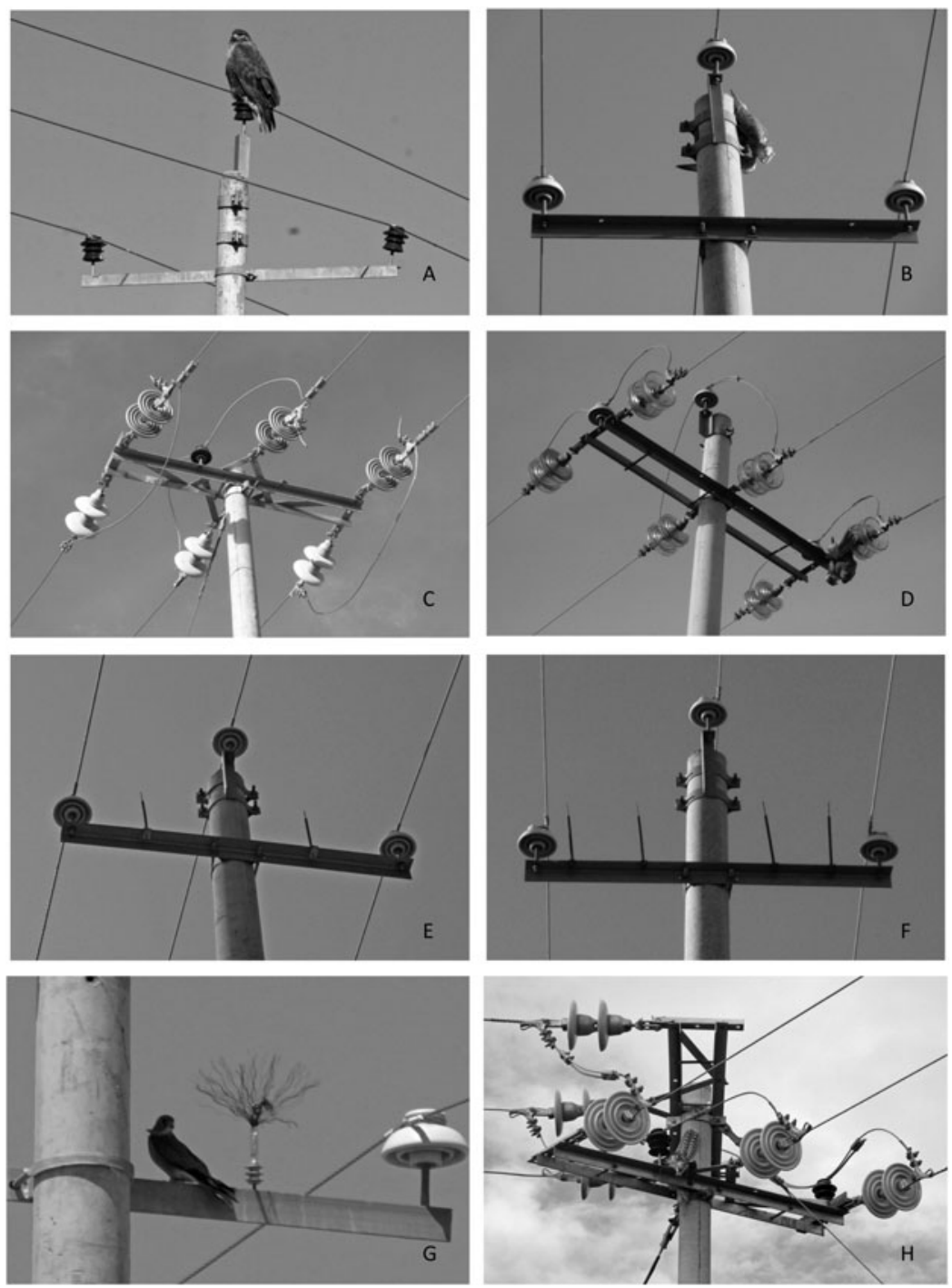

Figures 1a-h. A: line pole with pin insulators for each phase, Qinghai, China; Upland Buzzard perched on central $1^{\text {st }}$ phase pin insulator. B: line pole with pin insulators for each phase, Sukhbaatar Province, Mongolia; Saker Falcon electrocuted at Ist phase pin insulator. C: anchor pole with strain insulators and one jump wire over the crossarm on the central $1^{\text {st }}$ phase, Qinghai, China. D: anchor pole with strain insulators and jump wires over the crossarm on all three phases, Sukhbaatar Province, Mongolia; Saker Falcon electrocuted at $3^{\text {rd }}$ phase jump wire on crossarm. E: line pole fitted with two centrally-positioned, non-functional perch-deterrent spikes; Sukhbataar Province, Mongolia. F: line pole fitted with four, functional perch-deterrent spikes; Sukhbataar Province, Mongolia. G: line pole with centrally positioned perch deterrent device, Dornogovi Province, Mongolia; Common Kestrel perched on crossarm. H: anchor pole at a line deviation point requiring two braced crossarms and jump wires, Qinghai, China; electrocuted Saker Falcon next to a pin insulator. 
perch-deterrent devices placed centrally on the crossarm (Figure 1g). On 13 June 2010, a single search was made along a $14-\mathrm{km}$ stretch of a three-phase, $6 \mathrm{kV}$ distribution line running from the district centre of Adaatsag to the settlement of Sum Khokh Burd. This line was constructed in 2004 and comprised 133 poles; there were no perch deterrents fitted to the crossarms on this line.

\section{Line searches in Qinghai, China}

In Maduo County, Qinghai, we searched the entire lengths of two 1o kV electricity distribution lines running from an electricity substation at Madoi to the village of Huangheyaun on 20-21 June 2007 (summer survey) and from a substation at Huashixia to a coal mine near Miancaowan on 14 January 2008 (winter survey). Both lines travelled over open, undulating and treeless landscapes on the Qinghai-Tibetan Plateau. Poles and hardware were similar to the Mongolian lines above, except that the upright pin insulators were taller than those used on the Mongolian lines (Figure 1a). Also, some deviation angles on the line required the use of two double braced crossarms on a single pole with jump wires passing from one to the other for all three phases (Figure $1 \mathrm{~h}$ ). The $16 \mathrm{~km}$ Madoi-Huangheyuan distribution line comprised 200 line poles and 19 anchor poles, whilst the $17 \mathrm{~km}$ Huashixia-Miancaowan distribution line comprised 239 line poles and 17 anchor poles.

\section{Data collection}

During each line search, we checked the base of all electricity poles once for carcasses and feather remains of birds. Many carcasses showed obvious signs of electrocution with burn marks on the feet, legs and feathers. We assumed that all carcasses found below poles had died from electrocution and we found no evidence of any other alternative cause of death. We made an assessment of the how long the carcasses had lain on the ground from the state of decomposition or the condition of the feather remains if the carcass had been removed. In Mongolia, from May to September, carcass decomposition by scavenging beetles was very rapid, with most of the flesh removed from the body of medium-sized raptors such as Saker Falcons and Upland Buzzards Buteo hemilasius within a few days (A. Dixon pers. obs.). We considered whole carcasses with feathers in good condition but where the body flesh had been eaten by beetles as recent electrocution events $(<I$ month old) when the ground under the carcass was stained dark brown by beetle excreta. If there was no evidence of beetle excreta underneath such carcasses they were considered to be $>$ I month old. We were not able to determine removal rates of carcasses by scavengers during our single-visit searches but in some cases we only found a large number ( $>50)$ of fresh-looking feathers that showed no obvious weathering, and in these circumstances we assumed that the bird had been electrocuted within one month and the carcase had been removed by a scavenger.

For power lines surveyed in Sukhbaatar Province, Mongolia and in Qinghai, China we collected data on the type of pole where we found electrocuted birds (i.e. anchor or line pole) and whether or not the poles had been equipped with perch-deflector spikes. However, this information was not recorded for the two lines surveyed in Dundgovi Province, Mongolia, thus we were unable to examine electrocution in relation to pole type or the presence of perch deflectors for these lines.

\section{Data analysis}

In our analysis, we have only included raptors that were assessed to have been electrocuted within one month of our searches. We used a $\chi^{2}$ test to compare electrocution events at poles with different hardware configurations and perch deterrents. We present data in terms of the number of carcasses found, the number per $10 \mathrm{~km}$ of power line and per 100 poles, so that comparison can be made between the different lines in our study and with other studies that report electrocution rates in these formats. 


\section{Results}

The opportunistic surveys reported here did not allow us to undertake the repeated visits necessary to assess mortality rate estimates per unit of time. However, all carcasses were judged to be less than one month old, thus the figures presented in Table 1 can be regarded as a broad estimate of 'monthly mortality rates' at different seasons with the caveat that carcass age assessment is subjective and that we do not have good measures of carcass removal rates.

\section{Electrocution in Mongolia}

During our spring search we found the remains of 41 raptors that had been electrocuted along the Uulbayan-Monkhkhaan line (Table 1). Species included Black Kite Milvus migrans, Common Buzzard Buteo buteo and Northern Goshawk Accipiter gentilis, which are all migrants that do not breed locally. In contrast, we only found one non-resident migratory species, a Greater Spotted Eagle Aquila clanga, among 25 electrocuted raptors during our autumn search (Table 2). We found 45 electrocuted raptors of four resident species, during our winter search.

The number of remains found during the spring, autumn and winter totalled $7.3,4.5$ and 8.0 carcasses per 1o km respectively, though we do not know how many carcasses had been removed by scavengers as some may have been removed without leaving feather remains. The proportion of carcasses scavenged was much higher in winter, when $65 \%$ of carcasses had been removed leaving only feather remains, compared to $5 \%$ and $4 \%$ in spring and autumn respectively.

Electrocuted raptors were found under both anchor and line poles (Figure 2). Over the course of three searches we recorded the frequency of electrocution events during 91 visits to anchor poles that had jump wires over the crossarm on one $(n=59)$ or three phases $(n=32)$, and we found that significantly more raptors were electrocuted on the latter $\left(4.8 \% c f .22 .0 \% ; \chi^{2}=5.475\right.$, $\mathrm{df}=1, P=0.02)$. Over the course of 1,465 visits to line poles fitted with none ( $n=817)$, nonfunctional $(n=240)$ and functional $(n=408)$ perch-deterrent spikes, we found no significant difference in the number of carcasses found at poles with no perch-deterrent spikes and those with non-functional spikes placed centrally on the crossarm $\left(7.7 \% c f .10 .8 \% ; \chi^{2}=1.958, \mathrm{df}=1\right.$, $P=0.16)$, but there were significantly fewer carcasses at poles fitted with functional perch-deterrent spikes $(2.7 \%)$ than at those with none $\left(\chi^{2}=11.19, \mathrm{df}=1, P<0.001\right)$ and non-functional spikes $\left(\chi^{2}=17.10, \mathrm{df}=1, P<0.001\right)$.

In Dundgovi Province, we found the carcasses of 23 raptors that had been electrocuted along the Olziit-Khuld and Adaatsag-Sum Khokh Burd lines, $35 \%$ of which were non-resident species (Table 2).

\section{Electrocution in China}

On 20-21 June 2007 (summer search), we found the carcasses of 27 electrocuted raptors along the Madoi-Huangheyuan distribution line, comprising 23 Upland Buzzards, three Saker Falcons and a Raven Corvus corax (Table 3). We judged that these had died within a month, but we were unable to estimate the rate of carcase removal by scavengers and/or power line workers. The majority of electrocutions occurred at anchor poles and $63.2 \%(12 / 19)$ had electrocuted birds

Table 1 . Summary of power lines surveyed in this study.

\begin{tabular}{lllrll}
\hline Country & Province & LINE NAME & $\mathrm{kV}$ & Length $(\mathrm{km})$ & Pole \# \\
\hline Mongolia & Sukhbaatar & Uulbayan-Monkhkhaan & 15 & 56 & 528 \\
Mongolia & Dundgovi & Olziit-Khuld & 15 & 71 & 629 \\
Mongolia & Dundgovi & Adaatsag-Sum Koch Burd & 6 & 14 & 133 \\
China & Qinghai & Madoi-Huangheyaun & 10 & 16 & 219 \\
China & Qinghai & Huashixia-Miancaowan & 10 & 17 & 256 \\
\hline
\end{tabular}




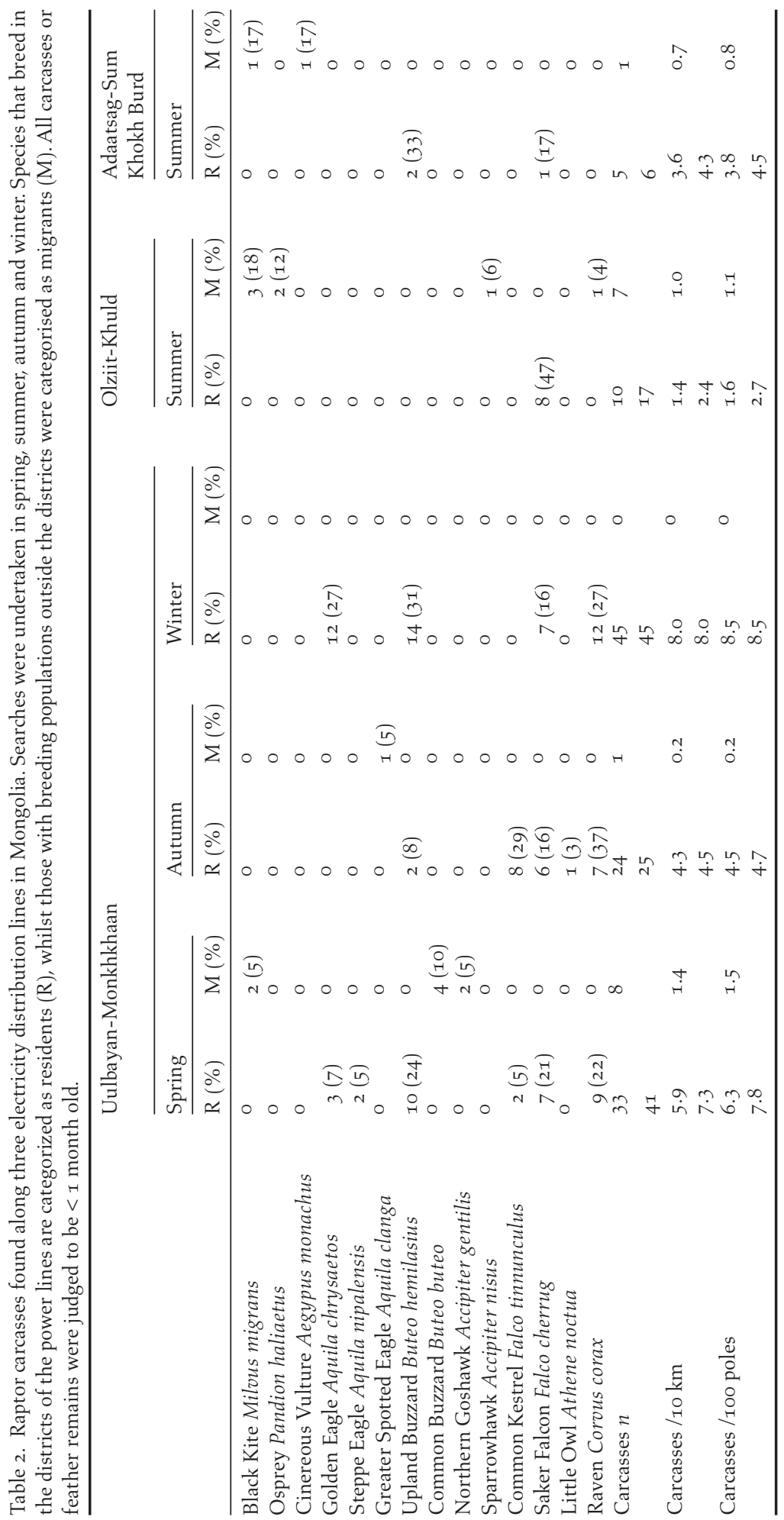




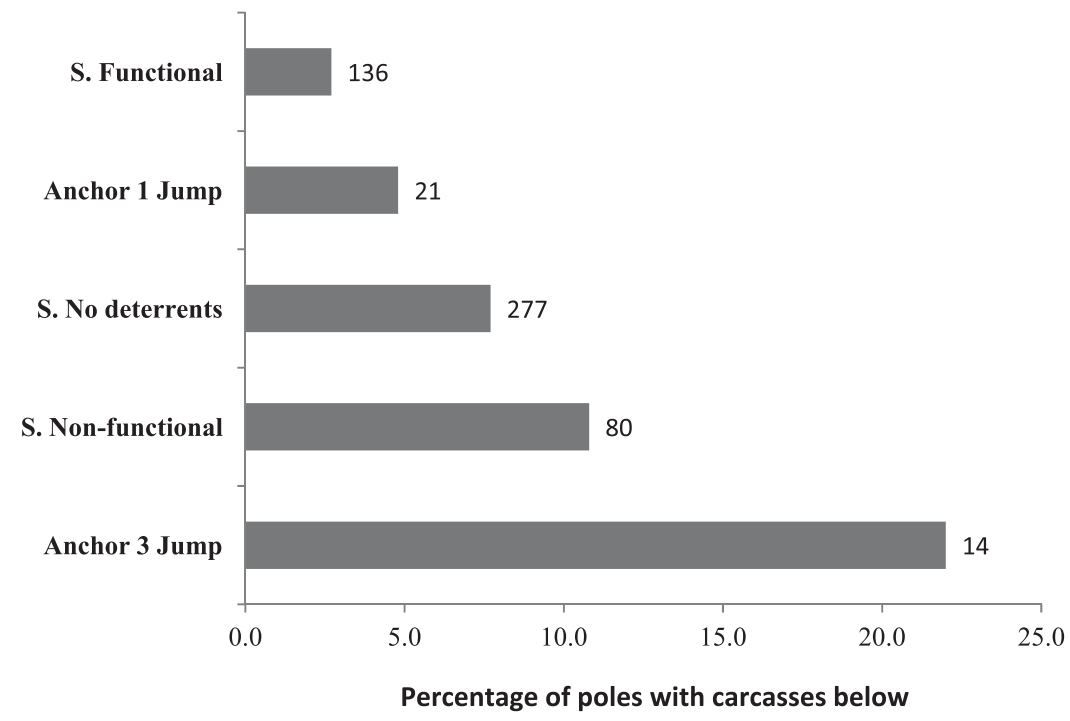

Figure 2. Percentage of poles with electrocuted raptors on the Uulbayan-Monkhkhaan line in Sukhbaatar Province, Mongolia. Values show the number of poles of each configuration on the line. Anchor pole types are (i) one jump wire over the crossarm on the central phase and (ii) three jump wires over the crossarm on all phases. Standard line pole types are crossarms with (i) no perch deterrent spikes, (ii) non-functional perch deterrent spikes and (iii) functional perch deterrent spikes.

below, whilst only $0.5 \%(\mathrm{I} / 200)$ of line poles had an electrocuted bird below. On this line, the high level of raptor electrocution events was attributable primarily to the presence of jump-wires over the crossarm of anchor poles.

On 14 January 2008 (winter search), we found the fresh feather remains of at least 1o different raptors, comprising six Upland Buzzards, two Saker Falcons and two Eagle Owls Bubo bubo and the whole, fresh carcass of a Common Kestrel Falco tinnunculus, together with the older, decomposed remains of a further 14 raptors (13 Upland Buzzards and a Saker Falcon) along the Huashixia-Miancaowan distribution line (Table 3). All remains, both recent and old, were found below anchor poles with overhead jump wires and none were found below line poles.

Table 3. Recent raptor remains (feathers or carcass) found below $10 \mathrm{kV}$ electricity distribution lines during surveys in summer (Madoi-Huangheyuan; $16 \mathrm{~km}, 219$ poles) and winter (Huashixia-Miancaowan; $17 \mathrm{~km}$, 256 poles) on the Qinghai-Tibetan Plateau, China.

\begin{tabular}{|c|c|c|c|c|}
\hline & \multicolumn{2}{|c|}{ Summer Survey } & \multicolumn{2}{|c|}{ Winter Survey } \\
\hline & Feathers & Carcass & Feathers & Carcass \\
\hline Upland Buzzard Buteo hemilasius & $\mathrm{o}$ & $23(85 \%)$ & $6(55 \%)$ & $\mathrm{O}$ \\
\hline Common Kestrel Falco tinnunculus & $\mathrm{O}$ & $\mathrm{O}$ & $\mathrm{O}$ & $I(9 \%)$ \\
\hline Saker Falcon Falco cherrug & o & $3(11 \%)$ & $2(18 \%)$ & o \\
\hline Eagle Owl Bubo bubo & $\mathrm{o}$ & 0 & $2(18 \%)$ & o \\
\hline Common Raven Corvus corax & $\mathrm{O}$ & $1(4 \%)$ & $\mathrm{O}$ & $\mathrm{o}$ \\
\hline Carcasses $n$ & 27 & & 11 & \\
\hline Carcasses /1o km & 16.9 & & 6.5 & \\
\hline Carcasses/100 poles & 12.3 & & $4 \cdot 3$ & \\
\hline
\end{tabular}




\section{Discussion}

The number and species of raptors electrocuted at a particular power line will reflect spatial and temporal variation in the composition of the raptor community and the propensity for a particular species to be electrocuted; larger species are at greater risk than smaller species and some taxa, such as harriers Circus spp., are less likely to utilise power poles as perch sites (Bevanger 1998, Lehman et al. 1999). The composition of the raptor community in Mongolia was different in spring/summer when $25 \%$ of electrocuted birds were non-resident migrants compared to autumn/winter, when such species comprised only $1 \%$ of the electrocuted birds. The higher frequency of feather remains in relation to whole carcasses found during the winter searches contrasted with our summer searches in Mongolia and China, indicating that carcass removal by scavengers varies at different times of year. The difference in carcass scavenging rates during summer and winter makes it difficult for us to meaningfully compare electrocution rates at different times of year. However, even though we lacked precise information on the period over which the raptors were electrocuted, it was apparent that a large number of raptors are electrocuted throughout the year at all the lines we surveyed. Despite the inherent limitations of opportunistic line surveys there is a need for dissemination of electrocution data in order to inform conservation management decisions relating to bird electrocution (Lehman et al. 2007).

Our line surveys in Qinghai revealed that anchor poles with jump wires over crossarms were especially dangerous for raptors, with few electrocution events recorded under line poles along the span of the distribution lines. In contrast, we found many electrocuted birds below line poles along the span of the Uulbayan-Monkkhaan line in Mongolia. In Qinghai, targeted mitigation focussed on anchor poles could potentially eliminate most raptor electrocution events on the lines we surveyed, whereas in Mongolia, even though anchor poles still killed disproportionately more raptors, such targeted mitigation would not reduce mortality levels to the same extent because many birds were killed at line poles, thus measures to reduce raptor electrocution events are required for all poles along the surveyed lines.

The reason why line poles in Mongolia posed a greater risk than their counterparts on the surveyed distribution lines in Qinghai is presumably related to differences in the configuration of the pin insulators rather than differences in bird behaviour in the two regions. In Qinghai, the energised cables were further from the metal crossarms and the top of the concrete pole than in Mongolia because the ceramic insulators were taller and they were fixed using longer pins (Figure $1 \mathrm{a}$ and $\mathrm{b}$ ).

Poles with perch deterrents placed in close proximity to the pin insulators on crossarms (i.e. the functional four-spike configuration) had significantly fewer electrocutions at our surveyed line in Sukhbaatar Province, Mongolia. This contradicts the findings of Harness et al. (2010) and Amartuvshin and Gombobaatar (2012); the former reported no effect of perch deterrent spikes on the same line, whilst the latter reported an increase in electrocution events with perch deterrents. However, Harness et al. (2010) did not distinguish between functional deterrent spikes placed near the insulators (four-spike configuration) and non-functional spikes placed centrally on the crossarm (two-spike configuration), whilst the data analysis of Amartuvshin and Gombobaatar (2012) is erroneous as the correlation between number of dead raptors and the number of spikes did not take into account the number of poles with different perch deterrent configurations. Aside from reconfiguring the hardware to a safe design on line poles (examples in APLIC 2006, Prinsen et al. 2011b), potential retrospective mitigation measures include fitting perch deterrents close to pin insulators on all crossarms or fitting covers over the pin insulators and adjacent exposed areas of the phase conductors.

Pole design has been shown to be the main factor accounting for variation in electrocution rates between power lines, and in order to maximise efficacy of remediation and optimise resource allocation it is advisable to undertake predictive modelling to identify the most important poles requiring mitigation (Tintó et al. 2010). In both Mongolia and China, jump wires over crossarms on anchor poles were especially dangerous for raptors. Electrocution can occur through direct contact with the energized jump wire (Figures $1 \mathrm{~d} \& \mathrm{~h}$ ). Consequently, mitigation can simply involve reconfiguration of the jump wires to pass under the crossarm, but if this is not possible at all three phases, especially the central first phase, mitigation could involve insulating the overhead jump wires and covering both the strain and pin insulators (examples in APLIC 2006, 
Prinsen et al. 2011b). However, insulation is best regarded as a temporary or complementary solution to reconfiguration and can be costly to undertake and to maintain (Tintó et al. 2010, Guil et al. 2011). With regard to mitigation and reconfiguration costs, the use of poles with safe designs at the outset is the most cost effective option when constructing new power distribution lines.

The globally threatened Saker Falcon is susceptible to electrocution on power distribution lines in the open steppe and plateau landscapes of Mongolia and Qinghai. This was reflected by the temporal stability in the number of Saker Falcons in our sample of electrocuted raptors obtained during different seasons in Sukhbaatar Province, Mongolia and in Qinghai, China. In Sukhbaatar Province, Mongolia there are five distribution lines (total $310 \mathrm{~km}$ ) known to have a high frequency of raptor electrocutions (S. Batkhuu pers. comm.). However, we do not know how many similar electricity distribution lines exist in other parts of Mongolia and Qinghai, but it is evident that the number is increasing with economic, industrial and social development. Consequently, mortality of Saker Falcons as a result of electrocution is also likely to increase in these two globally important bioregions for the species. Further work is required to determine if mortality rates due to electrocution have a significant impact on Saker Falcon breeding populations in the Mongolian steppe and the Qinghai-Tibetan Plateau.

\section{Acknowledgements}

This study was undertaken on behalf of and funded by the Environment Agency-Abu Dhabi (EAD). Surveys in Mongolia were conducted under a Memorandum of Understanding between EAD, the Ministry of Nature, Environment and Tourism (MNET) and International Wildlife Consultants Ltd. We thank HE M. Al Bowardi for his support and interest in this study. The following provided assistance in the field: C. Ashford, Batzul B., Hu B-W., Mei Y. and P. Stafford. S. Batkhuu (Sukhbaatar branch of the Eastern Energy System, Mongolia) and Shi Y. (Qinghai Electrical Power, China) provided background information on power distribution lines and raptor electrocution in their respective regions.

\section{References}

Amartuvshin, P. and Gombobaatar, S. (2012) The assessment of high risk utility lines and conservation of globally threatened pole nesting steppe raptors in Mongolia. Ornis Mongolica 1: 2-12.

Angelov, I., Hashim, I. and Oppel, S. (2013) Persistent electrocution mortality of Egyptian Vultures Neophron percnopterus over 28 years in East Africa. Bird Conserv. Internatn. 23: 1-6.

APLIC (2006) Suggested practices for avian protection on power lines: State of the art in 2006. Washington, DC \& Sacramento, CA: Edison Electric Institute, Avian Power Line Interaction Committee \& California Energy Commission.

Bevanger, K. (1998) Biological and conservation aspects of bird mortality caused by electricity power lines: a review. Biol. Conserv. 86: 67-76.

Boshoff, A. F., Minnie, J. C., Tambling, C. J. and Michael, M. D. (2011) The impact of power line-related mortality on the Cape Vulture Gyps coprotheres in a part of its range, with an emphasis on electrocution. Bird Conserv. Internatn. 21: 311-327.

Chaurey, A., Ranganathan, M. and Mohanty, P. (2004) Electricity access for geographically disadvantaged rural communities - technology and policy insights. Energ. Policy 32: 1693-1705.

Dixon, A. (2010) The problem of raptor electrocution at electricity distribution lines. Falco 37: 10-13.

Foster, V. and Briceño-Garmendia, C., eds. (2010) Africa's infrastructure: a time for transformation. Washington DC: International Bank for Reconstruction and Development/The World Bank.

Gombobaatar, S., Sumiya, D., Shagdarsuren, O., Potapov, E. and Fox, N. (2004) Saker Falcon (Falco cherrug milvipes Jerdon) mortality in Central Mongolia and population threats. Mongolian J. Biol. Sci. 2:13-21. 
Guil, F., Fernández-Olalla, M., Moreno-Opo, R., Mosqueda, I., Gómez, M. E., Aranda, A., Arredondo, A., Guzmán, J., Oria, J., González, L. M. and Margalida, A. (2011) Minimising mortality in endangered raptors due to power lines: the importance of spatial aggregation to optimize the application of mitigation measures. PLOS ONE 6(II): e28212. doi:10.1371/journal.pone.0028212

Hammons, T. J. (2011) Electricity infrastructure in Asian region and energy security problems. Pp. 415-450 in T. J. Hammons, ed. Electricity infrastructures in the global marketplace. InTech, www.intechopen.com.

Harness, R., Gombobaatar, S. and Yosef, R. (2008) Mongolian distribution power lines and raptor electrocutions. Pp. C1.1 - C1.6. in 2008 Rural Electric Power Conference April 27-30, Charleston, South Carolina. New York: IEEE.

Harness, R., Gombobaatar, S. and Amartuvshin, P. (2010) Evaluation of retrofitting measures in the Mongolia steppe. Fort Collins, Colorado: EDM International.

Lasch, U., Zerbe, S. and Lenk, M. (2010) Electrocution of raptors at power lines in Central Kazakhstan. Forest Ecol., Landscape and Nat. Conserv. 9: 95-100.

Lehman, R. N., Ansell, A. R., Garrett, M. G., Miller, A. D. and Olendorff, R. R. (1999) Suggested practices for raptor protection on power lines: the American story. Pp. 125159 in M. Ferrer and G. F. E. Janss, eds. Birds and power lines: collision, electrocution and breeding. Madrid, Spain: Quercus.

Lehman, R. N., Kennedy, P. L. and Savidge, J. A. (2007) The state of the art in raptor electrocution research: a global review. Biol. Conserv. 136: 159-174.

Mei, Y., Ma, M., Dixon, A. and Hu, B-W. (2008) Investigation of raptor electrocution along power lines in Western China. Chi. J. Zool. 43: 114-117. [In Chinese].

Prinsen, H. A. M., Boere, G. C., Píres, N. and Smallie, J. J. (2011a) Review of the conflict between migratory birds and electricity power grids in the African-Eurasian region. Bonn, Germany: CMS Technical Series No. XX, AEWA Technical Series No. XX.

Prinsen, H. A. M., Smallie, J. J., Boere, G. C. and Píres, N. (2011b) Guidelines on how to avoid or mitigate impact of electricity power grids on migratory birds in the African-Eurasian region. Bonn, Germany: CMS Technical Series No. XX, AEWA Technical Series No. XX.

Saltykov, A. V. (2012) Results of the scientific workshop "Problems of Bird Electrocution and Safety on Overhead Power Lines of Middle Voltage: Modern Scientific and Practice Experience" (Ulyanovsk, 10-11 November 2011), Russia. Raptors Conservation 24: 24-29.

Tintó, A., Real, J. and Manosa, S. (2010) Predicting and correcting electrocution of birds in Mediterranean areas. J. Wildl. Manage. 74: 1852-1862

\section{ANDREW DIXON*}

International Wildlife Consultants Ltd., PO Box 19, Carmarthen SA33 5 YL, UK.

\section{ROLLER MAMING}

Xinjiang Institute of Ecology and Geography, Chinese Academy of Sciences, 818 Beijing Road, Urumqi 830011, Xinjiang, China.

AMARKHUU GUNGA, GANKHUYAG PUREV-OCHIR, NYAMBAYAR BATBAYAR Wildlife Science and Conservation Center, Office 33, Undram Plaza, Bayanzurkh District, Ulaanbaatar 51, Mongolia.

${ }^{*}$ Author for correspondence; e-mail: falco@falcons.co.uk 